\title{
Design of a Wide-Bandwidth on-Chip Antenna for Uncooled Passive THz Imaging
}

\author{
Bernhard Klein $^{*(1)(3)}$, Thomas Morf ${ }^{(1)}$, Michel Despont ${ }^{(1)}$, Ute Drechsler ${ }^{(1)}$, Dan Corcos ${ }^{(2)}$, Noam Kaminski ${ }^{(2)}$, \\ Danny Elad $^{(2)}$, Lukas Kull ${ }^{(1)}$, Matthias Braendli ${ }^{(1)}$, Thomas Toifl ${ }^{(1)}$, Ronny Hahnel ${ }^{(3)}$ and Dirk Plettemeier ${ }^{(3)}$ \\ (1) IBM Research - Zurich, Säumerstrasse 4, 8803 Rüschlikon, Switzerland \\ Email:TMR@zurich.ibm.com \\ (2) IBM Research - Haifa, Haifa University Campus, Mount Carmel, Haifa, 31905, Israel \\ Email:DANCO@il.ibm.com \\ (3) Dresden University of Technology - Chair for RF Engineering, 01062 Dresden, Germany \\ Email: Bernhard.Klein@mailbox.tu-dresden.de
}

\begin{abstract}
The design of a broadband on-chip antenna for passive THz imaging in the frequency range of $0.6 \mathrm{THz}$ to $1.4 \mathrm{THz}$ is reported. The antenna design has to fulfill the requirements of the IBM CMOS process and the MEMS post CMOS processing. The antenna is coupled directly to the sensor, a MOSFET bolometer. Because of this direct coupling and the need for real time imaging, only extremely physically small antennas are feasible. Hence, typical broadband antennas like the toothed log-periodic antenna are not useable for this application and new antenna approaches have to be examined.
\end{abstract}

\section{INTRODUCTION}

There is a great interest in passive THz imaging as it opens new possibilities in security and medical applications [1, 2]. Most of the current research in passive THz imaging is done for cooled detectors. In contrast, our approach is expected to enable imaging at room temperature. The specified frequency range of $0.6 \mathrm{THz}$ to $1.4 \mathrm{THz}$ allows penetrating clothes. On the other hand, energy emission of objects at $300 \mathrm{~K}$ is very small making passive THz imaging very challenging.

A pixel in our approach consists of the antenna with termination resistors, a MOSFET, holding arms, and a frame. Below the pixel array a common reflector, built by the package, is positioned at a $60 \mu \mathrm{m}$ distance to the antennas.

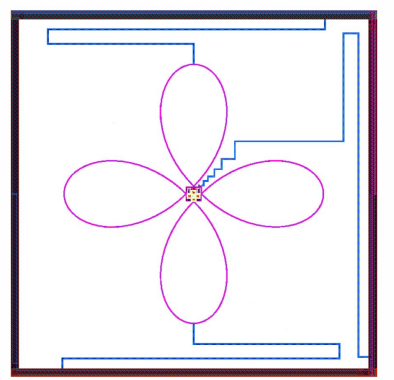

Fig. 1 Schematic of one pixel

The received signal is converted into heat by a termination resistor. A MOSFET, acting as a temperature sensor, is therefore indirectly detecting the $\mathrm{THz}$ signal [3]. As the heat has to stay at the sensor, a high thermal isolation between antenna/sensor and the rest of the chip has to be ensured. This is achieved by completely etching the surrounding silicon and by using long and narrow holding arms made of poly-silicon, connecting the antenna to the frame. The holding arms serve for the biasing of the MOSFET and for the read-out as well. To enable room temperature operation a MEMS post CMOS processing step after fabrication of the chip in the $180 \mathrm{~nm}$ CMOS SOI technology is required. Design rules of the MEMS process requirement and the specification for real time imaging result in constraints for the antenna design.

To achieve real time images, the thermal mass of the whole pixel has to be minimized. As the antenna is coupled directly to the sensor, it is part of the overall thermal mass. Hence, physically big antennas are not appropriate for this 
approach. The thermal mass of the antenna and in consequence the physical area has to be small. The height of the layers in the used CMOS SOI process is fixed. These requirements lead to very fragile antenna structures.

\section{MEMS POST CMOS PROCESSING STEP}

Due to the specification of passive THz imaging at room temperature and the small energy emission of objects in the targeted frequency range, a MEMS post-processing step is required. Other approaches which operate at temperatures below $4 \mathrm{~K}$ use the superconducting effect of metal and hence, a MEMS post CMOS processing step is not necessary. Fig. 2 describes the MEMS post-process [4].
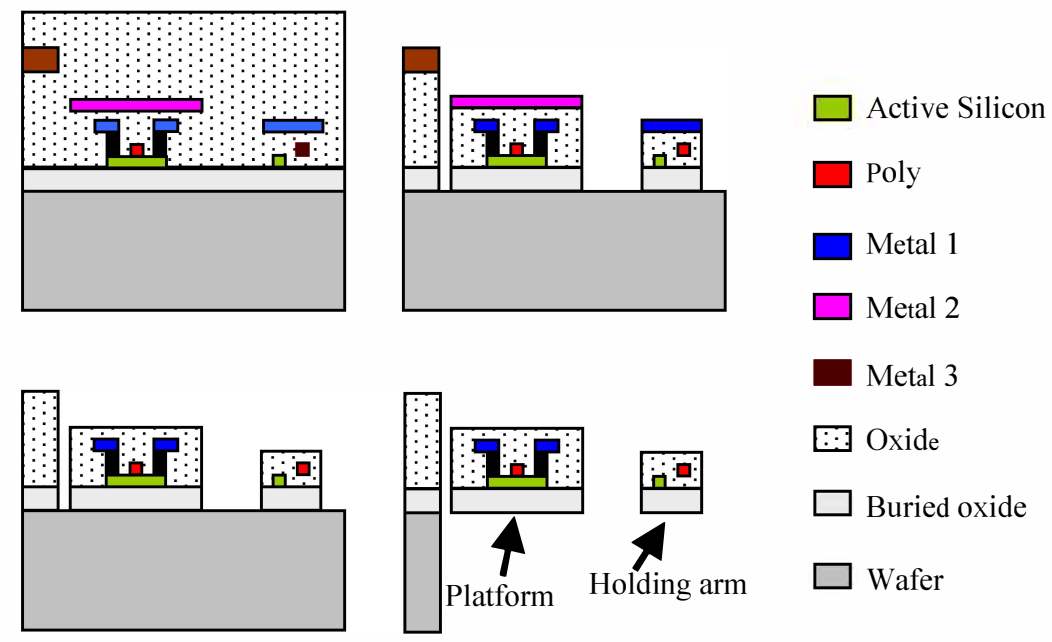

Fig. 2 Post CMOS MEMS process [4]

In the top left, the cross-section after the standard CMOS SOI process is shown. In the top right, all silicon oxide is etched away from the front. Metal layers serve as etch stops. The next step is front-side etching of the topmost metal layer, shown in the bottom left. Last step is a backside etching of the wafer substrate (bottom right).

Accordingly, the antenna, the holding arms, and the platform have to be protected by an on-chip mask, which is the metal layer above the structures (antenna, holding arm, and platform). These on-chip masks have to be drawn with a small overlap (about $200 \mathrm{~nm}$ ), so the antenna structures are protected by silicon oxide after removing the mask-metal. Hence, the thermal mass of the antenna is not only defined by the size of the antenna but also by the volume of the surrounding silicon oxide. Therefore, a physically minimized antenna size is required. A cloverleaf shaped antenna after the MEMS post-process is shown in Fig. 3.

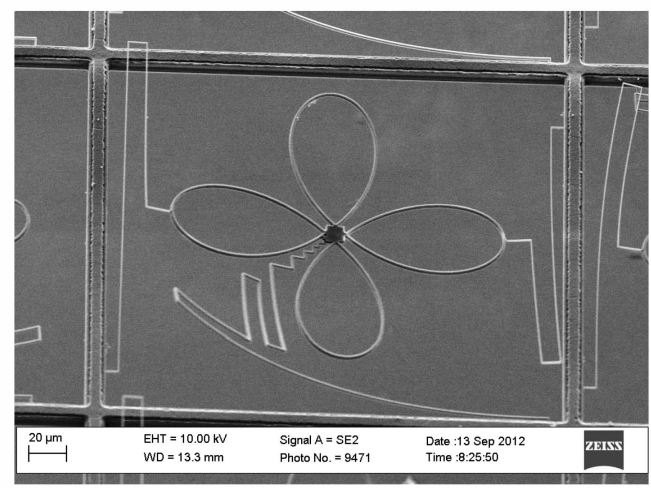

Fig. 3 SEM picture of the processed cloverleaf antenna (Pixel size $250 \mu \mathrm{m} \times 250 \mu \mathrm{m}$ )

The two shorter holding arms are oxide only and support the mechanical stability of the antenna structure. The whole pixel is surrounded by the frame. Besides the antenna, the holding arms, the central platform, and the frame, everything is etched off. 


\section{ANTENNA SIMULATION}

Due to the bandwidth requirements and the thermal mass problems, most of the standard broadband antennas are not feasible for our approach. We propose a cloverleaf shaped antenna. HFSS by Ansys [5] was used for the simulations. The HFSS model of the antenna is shown in Fig. 4.

The overall dimensions of a pixel including the frame are $250 \mu \mathrm{m} \times 250 \mu \mathrm{m}$. The frame has a width of $10 \mu \mathrm{m}$ and is cut into four pieces, to avoid resonances. There is one active holding arm (red/green - zig-zag) built of an active and a poly-silicon line. The holding arms in violet are oxide holding arms, which support the mechanical stability of the antenna structure. The antenna is made out of a Metal 1 and has a linewidth of $260 \mathrm{~nm}$.

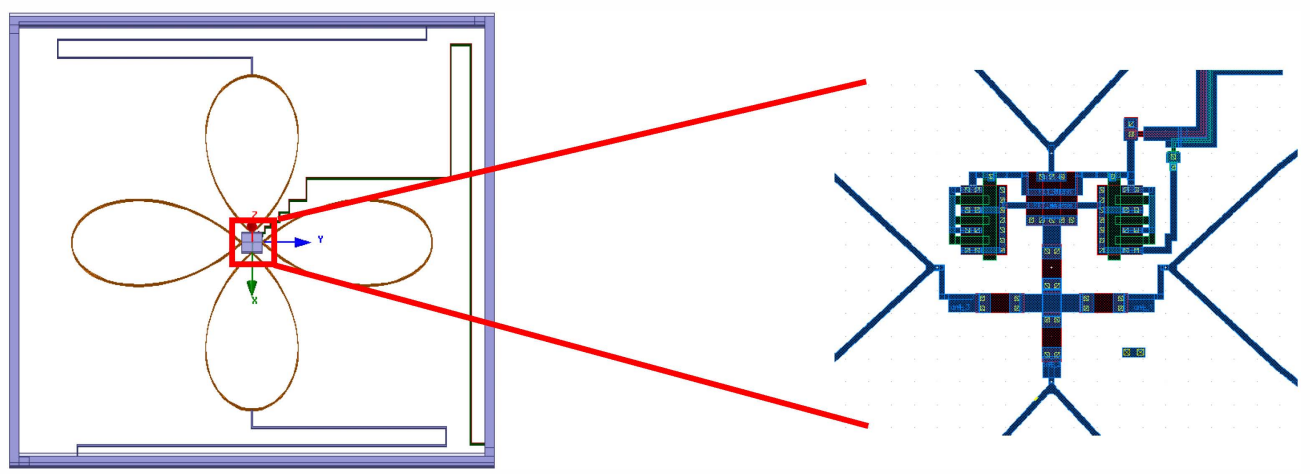

Fig. 4 Antenna model. Left: Simulation model of the whole pixel, right: Layout of the platform in the center including the sensor

In this HFSS simulation model not all layers are represented, as this would result in too high computational effort. Metal, silicon oxide, and the silicon nitride layers next to the antenna are part of the model, but thin layers like the liners around the metals are considered to be negligible. The antenna was simulated with two ports instead of the two physical termination resistors.

The two opposite antenna arms are physically connected with a termination resistor of $200 \Omega$ each, as is shown in Fig. 4. Two MOSFETs are acting as temperature sensors inside the platform.

Directivity vs. frequency is shown in Fig. 5. The directivity plotted here, is defined in normal direction to the antenna plane $\left(\theta=0^{\circ}\right)$.

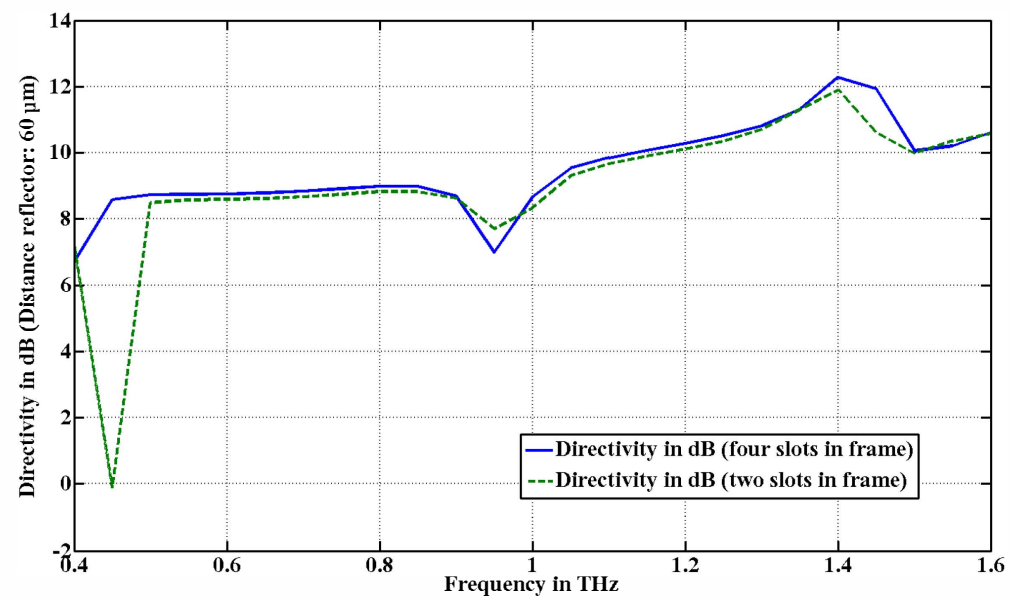

Fig. 5 Directivity vs. frequency plot of the cloverleaf antenna including the reflector

To suppress resonances in the frame, which create a dip in the directivity, the frame was cut in sections. The dashed curve represents the directivity in $\mathrm{dB}$, when the pixel frame is cut in two pieces and the continuous one represents the directivity in $\mathrm{dB}$, when the frame is cut in four pieces.

As frequencies near $500 \mathrm{GHz}$ will be strongly attenuated by water absorption in air (e.g. humidity), the dip in the green curve is not a problem. Above $1400 \mathrm{GHz}$, the directivity becomes too small to obtain good area efficiencies. The effective area is proportional to the wavelength squared, which has a greater influence at higher frequencies than the 
approximately flat directivity could compensate for. A high area efficiency, which is connected to the directivity, is needed. Values for the area efficiency above one are counterproductive, since then energy intended for neighboring pixels would be collected. The small improvement of the frame with two slots can be noticed at $950 \mathrm{GHz}$. Here the dip in the green (dashed) curve is less than the dip in the blue one (solid).

Fig. 6 shows the directivity pattern of the antenna (with a two slot frame) for three different frequencies $(600 \mathrm{GHz}-$ $\mathrm{red} /$ continuous, $1000 \mathrm{GHz}$ - blue/dashed, $1400 \mathrm{GHz}$ - green/dash-dot) and for the angle $\varphi=0$ deg. For the normal direction, the maximum directivity is $12.2 \mathrm{~dB}$ at $1400 \mathrm{GHz}$, which corresponds to an area efficiency of approximately 0.97. At $600 \mathrm{GHz}$, the maximum directivity is $8.7 \mathrm{~dB}$ and at $1000 \mathrm{GHz}$ it is $8.5 \mathrm{~dB}$. The half-power beam width at $1400 \mathrm{GHz}$ is approximately $40 \mathrm{deg}$ and at $600 \mathrm{GHz}$ and $1000 \mathrm{GHz}$ it is approximately $80 \mathrm{deg}$.

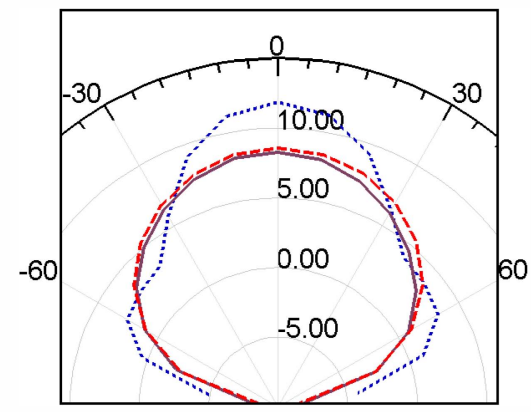

Fig. 6 Directivity pattern of cloverleaf antenna (solid - $1000 \mathrm{GHz}$, dashed - $600 \mathrm{GHz}$, dotted - $1400 \mathrm{GHz}$ )

Directivity is plotted rather than matched gain since matching is a smaller issue for this receiving antenna. Energy dissipated in the antenna will heat up the antenna and heat from the antenna arms will reach the sensor by heat conduction as well. A reflected signal due to mismatching is expected to mostly heat the antenna and is therefore not completely lost.

A $60 \mu \mathrm{m}$ distance of the reflector to the antenna corresponds to a quarter wavelength at a frequency of $1.25 \mathrm{THz}$. Hence, it acts constructively for the higher frequencies and destructively for the lower ones. The decrease in directivity for lower frequencies results in an area efficiency slightly smaller than one, which is acceptable. However, the area efficiency for the higher frequencies is increased.

\section{CONCLUSION}

The cloverleaf shaped antenna is a good antenna design for a passive and uncooled THz-imager. Together with a reflector positioned $60 \mu \mathrm{m}$ below the antenna good directivity values in the frequency range of $0.6 \mathrm{THz}$ to $1.4 \mathrm{THz}$ can be obtained. This results in good area efficiency over the whole frequency range.

The required MEMS post-process step limits the possible design variations and leads to a demanding manufacturing, as the cloverleaf shaped antenna consists of very soft and fragile structures.

The simulations shown above are for single pixels. Simulations with neighbouring pixels including crosstalk are ongoing as well as first antenna pattern measurements at $655 \mathrm{GHz}$.

The research leading to these results has received funding from the European Union Seventh Framework Program (FP7/2007-2013) under grant agreement $\mathrm{n}^{\circ}[288442]$.

\section{REFERENCES}

[1] L. Gitelman, S. Stolyarova, S. Bar-Lev, Z. Gutman, Y. Ochana, and Yael Nemirovsky, "CMOS-SOI-MEMS Transistor for Uncooled IR Imaging”, IEEE TRANSACTIONS ON ELECTRON DEVICES, VOL. 56, NO. 9, Sept. 2009

[2] Y. Lee, "Principle of Teraherz Science and Technology, Springer 2008

[3] D. Corcos, I. Brouk, M. Malits, A. Svetlitza, S. Stolyarova, A. Abramovich, E. Farbe, N. Bachar, D. Elad and Y. Nemirovsky, "The TeraMOS Sensor for Monolithic Passive THz Imagers", PROC. COMCAS, 2011.

[4] T. Morf et al, "Room-temperature THz imaging based on antenna-coupled MOSFET Bolometer", Proc. IEEE MEMS 2013, January 20-23, Taipei, Taiwan Conference (in press)

[5] Ansys: http://www.ansys.com/Products/Simulation+Technology/Electromagnetics/High-

Performance+Electronic+Design/ANSYS+HFSS 